\title{
PARABOLIC PROBLEMS WITH DYNAMICAL BOUNDARY CONDITIONS IN PERFORATED MEDIA
}

\author{
C. TIMOFTE \\ Department of Mathematics, Faculty of Physics, University of Bucharest \\ P.O. Box MG-11, Bucharest-Magurele, Romania \\ E-mail: claudiatimofte@hotmail.com
}

Received August 5 2003; revised December 42003

\begin{abstract}
The asymptotic behavior of the solution of a parabolic dynamical boundary-value problem in a periodically perforated domain is analyzed. The perforations, which are identical and periodically distributed, are of size $\varepsilon$. In the perforated domain we consider a heat equation, with a Dirichlet condition on the exterior boundary and a dynamical boundary condition on the surface of the holes. The limit equation, as $\varepsilon \rightarrow 0$, is a heat equation with extra-terms coming from the influence of the non-homogeneous dynamical boundary condition.
\end{abstract}

Key words: homogenization, energy method, dynamical boundary-value problems

\section{INTRODUCTION AND FORMULATION OF THE PROBLEM}

The aim of this paper is to study the asymptotic behavior of the solution of a parabolic dynamical boundary-value problem in a periodically perforated domain. Such problems, although not too widely considered in the literature, are very natural in many mathematical models as partially saturated flows in porous media, heat transfer in a solid in contact with a moving fluid, diffusion phenomena in porous media (see [2] and [4] and the references therein).

Let $\Omega$ be a fixed bounded open subset in $\mathbb{R}^{N}$ and let us perforate it by holes. As a result, we obtain an open set $\Omega^{\varepsilon}$, which will be referred to as being the perforated domain; $\varepsilon$ represents a small parameter, related to the characteristic size of the perforations. We shall deal with the case in which the perforations (holes) are identical and periodically distributed and their size is of the order of $\varepsilon$. In the perforated domain we consider a heat equation, with a Dirichlet condition on the exterior boundary and a dynamical boundary 
condition on the surface of the holes.

Our main motivation is to study the asymptotic behavior, as $\varepsilon \rightarrow 0$, of the solution $u^{\varepsilon}$ of the following dynamical boundary-value problem:

$$
\left\{\begin{array}{l}
\frac{\partial u^{\varepsilon}}{\partial t}-\Delta u^{\varepsilon}=f(t, x), \text { in } \Omega^{\varepsilon} \times(0, T) \\
\frac{\partial u^{\varepsilon}}{\partial n}+\varepsilon \frac{\partial u^{\varepsilon}}{\partial t}=\varepsilon g(t, x), \text { on } \partial F^{\varepsilon} \times(0, T) \\
u^{\varepsilon}(0, x)=u^{0}(x), \text { in } \Omega^{\varepsilon} \\
u^{\varepsilon}(0, x)=v^{0}(x), \text { on } \partial F^{\varepsilon} \\
u^{\varepsilon}=0, \text { on } \partial \Omega \times(0, T)
\end{array}\right.
$$

Here, $f \in L^{2}\left(0, T, L^{2}(\Omega)\right), g \in L^{2}\left(0, T, H_{0}^{1}(\Omega)\right), u^{0} \in L^{2}(\Omega), v^{0} \in L^{2}\left(\partial F^{\varepsilon}\right)$, $[0, T]$ is the time interval of interest and $\partial F^{\varepsilon}$ is the boundary of the holes. Also, let us notice that in the second equation of (1.1) the contribution of the dynamical part of our boundary condition on the surface of the holes and the contribution of the external force acting on the surface of the perforations are well-balanced by the presence of the parameter $\varepsilon$.

We shall prove in Section 4 that there exists an extension $\tilde{u}^{\varepsilon}$ of $u^{\varepsilon}$ into all $\Omega \times(0, T)$ such that $\tilde{u}^{\varepsilon} \rightarrow u$ strongly in $L^{2}\left(0, T ; L^{2}(\Omega)\right)$ and $u$ is the unique solution of some boundary value problem for PDE (the macromodel).

The plan of the paper is the following one: in Section 2 we introduce some useful notations and assumptions. In Section 3 we give the main convergence result of this paper, i. e. the macromodel. For obtaining this macromodel, we need some preliminary results, which are given in Section 4 . The last section is devoted to the proof of some a priori estimates, independent of $\varepsilon$, for the solution of the micromodel and to the proof of the convergence result.

The method we follow in this paper is the so-called energy method of $\mathrm{L}$. Tartar (see, for instance, $[15 ; 20]$ ). Using the same method, problems similar to this one have been considered by many authors. Among others, let us mention the papers of D. Cioranescu and P. Donato [6; 7], C. Conca and P. Donato [12], C. Conca, J.I. Díaz and C. Timofte [11], C. Conca, F. Murat and C. Timofte [13]. The homogenization of Laplace and Poisson equations in perforated domains with holes of the same size as the period and with homogeneous Dirichlet conditions on the surface of the holes and on the exterior boundary of the domain was treated in [6]. The same problem, but with homogeneous Neumann boundary conditions on the holes, was treated in [9]. For the non-homogeneous case, we can refer to [12]. The homogenization of the Poisson equation (or even a more general elliptic equation) with non-homogeneous Fourier boundary conditions on surface of the holes has been treated in $[6 ; 7]$.

Apart from the energy method, in the last three decades several alternative methods have been developed for studying such problems. Among them, let us 
mention the $\Gamma$-convergence of integral functionals, originated in [14] (see, also, $[18])$, the theory of two-scale convergence $([1 ; 22])$, the method of two-scale asymptotic expansion and, also, averaging methods ([3; 19]).

\section{NOTATION AND ASSUMPTIONS}

Let $\Omega$ be a bounded connected open set in $\mathbb{R}^{N}(N \geq 2)$, with boundary $\partial \Omega$ of class $C^{2}$ and let $[0, T]$ be the time interval of interest.

Let $Y=\left[0, l_{1}\left[\times \ldots \times\left[0, l_{N}\left[\right.\right.\right.\right.$ be the representative cell in $\mathbb{R}^{N}$ and $F$ an open subset of $Y$ with boundary $\partial F$ of class $C^{2}$, such that $\bar{F} \subset Y$.

We shall denote by $F(\varepsilon, \mathbf{k})$ the translated image of $\varepsilon F$ by the vector $\varepsilon \mathbf{k l}$, $\mathbf{k} \in \mathbb{Z}^{N}, \mathbf{k l}=\left(k_{1} l_{1}, \ldots, k_{N} l_{N}\right)$.

$$
F(\varepsilon, \mathbf{k})=\varepsilon(\mathbf{k} \mathbf{l}+F) .
$$

Also, we shall denote by $F^{\varepsilon}$ the set of all the holes contained in $\Omega$. So

$$
F^{\varepsilon}=\bigcup_{k \in K}\{F(\varepsilon, \mathbf{k}) \mid \bar{F}(\varepsilon, \mathbf{k}) \subset \Omega\} .
$$

Let $\Omega^{\varepsilon}=\Omega \backslash \overline{F^{\varepsilon}}$. (see Figure 1).
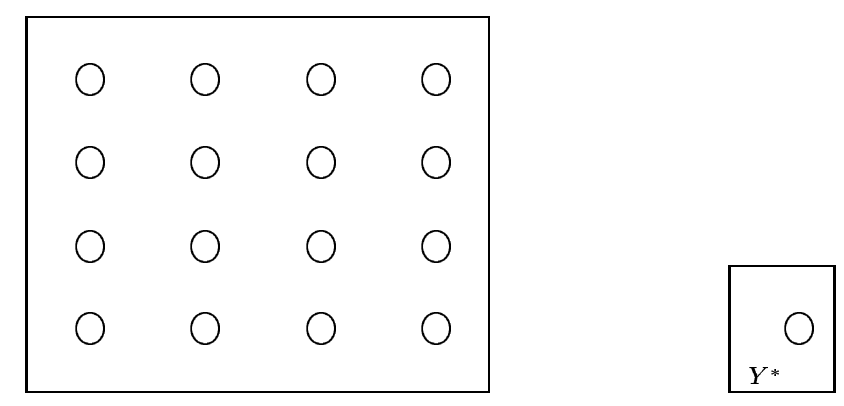

Figure 1. Definition of the regions.

Hence, $\Omega^{\varepsilon}$ is a periodically perforated domain with holes of the same size as the period. Let us remark that the holes do not intersect the boundary $\partial \Omega$.

We shall use the following notations:

$$
Y^{*}=Y \backslash \bar{F}, \quad \theta=\frac{\left|Y^{*}\right|}{|Y|} .
$$

Also, we shall denote by $\chi^{\varepsilon}$ the characteristic function of the domain $\Omega^{\varepsilon}$. 
Let us introduce the following usual function spaces and norms:

$$
\begin{aligned}
& H=L^{2}(\Omega),(u, v)_{\Omega}=\int_{\Omega} u v d x,\|u\|_{\Omega}^{2}=(u, u)_{\Omega}, \\
& \mathcal{H}=L^{2}(0, T ; H),(u, v)_{\Omega, T}=\int_{0}^{T}(u(t), v(t))_{\Omega} d t,\|u\|_{\Omega, T}^{2}=(u, u)_{\Omega, T}, \\
& V=H^{1}(\Omega),(u, v)_{V}=(u, v)_{\Omega}+(\nabla u, \nabla v)_{\Omega}, \\
& \mathcal{V}=L^{2}(0, T ; V),(u, v)_{\mathcal{V}}=\int_{0}^{T}(u(t), v(t))_{V} d t .
\end{aligned}
$$

\section{THE CONVERGENCE RESULT}

As we shall see in Section 4 , for $f \in L^{2}\left(0, T, L^{2}(\Omega)\right), g \in L^{2}\left(0, T, H_{0}^{1}(\Omega)\right)$, $u^{0} \in L^{2}(\Omega)$ and $v^{0} \in L^{2}\left(\partial F^{\varepsilon}\right)$, there exists a unique solution $u^{\varepsilon}$ of the problem (1.1).

The main result of this paper is the following one:

Theorem 3.1. Let $u^{\varepsilon}$ be the unique solution of the problem (1.1). Then, there exists an extension $\tilde{u}^{\varepsilon}$ of $u^{\varepsilon}$ into all $\Omega \times(0, T)$ such that $\tilde{u}^{\varepsilon} \rightarrow u$ strongly in $\mathcal{H}$ and $u$ is the unique solution of the following system (the macromodel):

$$
\left\{\begin{array}{l}
\left(\frac{\left|Y^{*}\right|}{|Y|}+\frac{|\partial F|}{|Y|}\right) \frac{\partial u}{\partial t}-\nabla(Q \nabla u)=\frac{\left|Y^{*}\right|}{|Y|} f+\frac{|\partial F|}{|Y|} g, \text { in } \Omega \times(0, T) \\
u=0, \text { on } \partial \Omega \times(0, T) \\
u(0, x)=u^{0}(x), \text { in } \Omega
\end{array}\right.
$$

where $Q=\left(q_{i j}\right)$ is the classical homogenized matrix whose entries are defined as

$$
q_{i j}=\frac{\left|Y^{*}\right|}{|Y|} \delta_{i j}-\frac{1}{|Y|} \int_{Y^{*}} \frac{\partial \eta_{j}}{\partial y_{i}} d y
$$

in terms of the functions $\eta_{j}$, solutions of the system

$$
\left\{\begin{array}{l}
-\Delta \eta_{j}=0, \text { in } Y^{*} \\
\partial\left(\eta_{j}-y_{j}\right) / \partial n=0, \text { on } \partial F \\
\eta_{j} \text { is } Y-\text { periodic }
\end{array}\right.
$$

here $y_{j}$ being local coordinates in $Y^{*}$.

Thus, in the limit, when $\varepsilon \rightarrow 0$, we get a classical constant coefficient heat equation, with a Dirichlet boundary condition, with a non-homogeneous right-hand term and with a constant (due to the periodicity) extra-term in 
front of the time derivative, coming from the well-balanced contribution of the dynamical part of our boundary condition on the surface of the holes.

Remark 3.1. The weak formulation of the problem (3.1) is:

Find $u \in L^{2}\left(0, T ; H_{0}^{1}(\Omega)\right) \cap C\left([0, T] ; L^{2}(\Omega)\right), u(0)=u^{0}$, such that

$$
\begin{aligned}
-\left(\frac{\left|Y^{*}\right|}{|Y|}+\frac{|\partial F|}{|Y|}\right)\left(u, \frac{\partial \varphi}{\partial t}\right)_{\Omega, T} & +(Q \nabla u, \nabla \varphi)_{\Omega, T} \\
& =\frac{\left|Y^{*}\right|}{|Y|}(f, \varphi)_{\Omega, T}+\frac{|\partial F|}{|Y|}(g, \varphi)_{\Omega, T},
\end{aligned}
$$

for any $\varphi \in \mathcal{D}=C_{0}^{\infty}((0, T) \times \Omega)$, or, equivalently:

$$
\begin{gathered}
-\frac{\left|Y^{*}\right|}{|Y|}\left(\int_{0}^{T}(u, \varphi)_{\Omega} \psi^{\prime}(s) d s+(u(0), \varphi)_{\Omega} \psi(0)\right)-\frac{|\partial F|}{|Y|} \int_{0}^{T}(u, \varphi)_{\Omega} \psi^{\prime}(s) d s \\
-\frac{|\partial F|}{|Y|}(u(0), \varphi)_{\Omega} \psi(0)+\int_{0}^{T}(Q \nabla u, \nabla \varphi)_{\Omega} \psi(s) d s \\
\quad=\frac{\left|Y^{*}\right|}{|Y|} \int_{0}^{T}(f, \varphi)_{\Omega} \psi(s) d s+\frac{|\partial F|}{|Y|} \int_{0}^{T}(g, \varphi)_{\Omega} \psi(s) d s, \quad \text { (3.4) }
\end{gathered}
$$

for any $\varphi \in H_{0}^{1}(\Omega), \psi \in C^{\infty}([0, T]), \psi(T)=0, \psi(0) \neq 0$.

Remark 3.2. There is one and only one solution of the weak macromodel problem.

\section{PRELIMINARY RESULTS}

\subsection{The Existence Result}

In order to obtain the existence and the uniqueness of a solution of problem (1.1), we shall make use of the following general result for abstract parabolic equations, due to J.L. Lions (see [5] and [16]).

Theorem 4.1. Let $\boldsymbol{H}$ be a Hilbert space, with scalar product (, ) and norm $|\cdot|$. We shall identify $\boldsymbol{H}$ with its dual. Let $\boldsymbol{V}$ be another Hilbert space, with norm $\|$.$\| . Suppose that \boldsymbol{V} \subset \boldsymbol{H}$, with continuous and dense embedding, such that $\boldsymbol{V} \subset \boldsymbol{H} \subset \boldsymbol{V}^{\prime}$. Let $0<T<\infty$ and $a: \boldsymbol{V} \times \boldsymbol{V} \rightarrow \mathbb{R}$ be a continuous and coercive bilinear form. For every $f \in L^{2}\left(0, T ; \boldsymbol{V}^{\prime}\right)$ and $u_{0} \in \boldsymbol{H}$, there exists a unique function $u$ such that $u \in L^{2}(0, T ; \mathbf{V}) \cap C([0, T] ; \mathbf{H}), \frac{d u}{d t} \in L^{2}\left(0, T ; \mathbf{V}^{\prime}\right)$ and

$$
\left\{\begin{array}{l}
\left\langle\frac{d u}{d t}(t), v\right\rangle+a(u(t), v)=\langle f(t), v\rangle, \text { a.e. } t \in[0, T], \forall v \in \mathbf{V}, \\
u(0)=u_{0}
\end{array}\right.
$$


Here, $\langle$,$\rangle denotes the duality between \boldsymbol{V}^{\prime}$ and $\boldsymbol{V}$. Also, one has the estimates:

$$
\begin{aligned}
& \|u\|_{L^{2}(0, T ; \mathbf{V})} \leq C\left(\|f\|_{L^{2}\left(0, T ; \mathbf{V}^{\prime}\right)}+\left\|u_{0}\right\|_{\mathbf{H}}\right), \\
& \left\|\frac{\partial u}{\partial t}\right\|_{L^{2}\left(0, T ; \mathbf{V}^{\prime}\right)} \leq C\left(\|u\|_{L^{2}(0, T ; \mathbf{V})}+1\right),
\end{aligned}
$$

with constants $C$ depending on $T$.

In our case, we choose $\mathbf{H}=L^{2}\left(\Omega^{\varepsilon}\right) \times L_{\partial \Omega}^{2}\left(\partial \Omega^{\varepsilon}\right)$,

$$
\begin{aligned}
& \mathbf{V}=\left\{\left(u_{1}, u_{2}\right) \in H_{\partial \Omega}^{1}\left(\Omega^{\varepsilon}\right) \times H_{\partial \Omega}^{1 / 2}\left(\partial \Omega^{\varepsilon}\right) / u_{2}=\gamma\left(u_{1}\right)\right\} \\
& a(u, v)=\frac{1}{\varepsilon} \int_{\Omega^{\varepsilon}} \nabla u_{1} \cdot \nabla v_{1} d x, \quad u_{0}=\left(u^{0}, v^{0}\right),
\end{aligned}
$$

where $u=\left(u_{1}, u_{2}\right), v=\left(v_{1}, v_{2}\right)$. In what follows, we shall use the standard Sobolev spaces $H_{\partial \Omega}^{r}\left(\Omega^{\varepsilon}\right)$ and $H_{\partial \Omega}^{r}\left(\partial \Omega^{\varepsilon}\right)$, for $r \geq 0$, which are closed subspaces of $H^{r}\left(\Omega^{\varepsilon}\right)$ and $H^{r}\left(\partial \Omega^{\varepsilon}\right)$, respectively, and the subscript $\partial \Omega$ means that, respectively, traces or functions in $\partial \Omega^{\varepsilon}$, vanish on this part of the boundary of $\Omega^{\varepsilon}$. Let us notice that, in fact, we can consider the given $v^{0}$ as an element of $L_{\partial \Omega}^{2}\left(\partial \Omega^{\varepsilon}\right)$.

So, we get immediately from Theorem 4.1 the existence of a unique solution of the problem (1.1),

$$
\left(u^{\varepsilon}, \gamma\left(u^{\varepsilon}\right)\right) \in L^{2}(0, T ; \mathbf{V}) \cap C([0, T] ; \mathbf{H}), \frac{d}{d t}\left(u^{\varepsilon}, \gamma\left(u^{\varepsilon}\right)\right) \in L^{2}\left((0, T) ; \mathbf{V}^{\prime}\right)
$$

with the initial conditions from (1.1).

The weak formulation of our problem is the following one:

Find $\left(u^{\varepsilon}, \gamma\left(u^{\varepsilon}\right)\right) \in L^{2}(0, T, \mathbf{V}) \cap C([0, T] ; \mathbf{H}), \frac{d}{d t}\left(u^{\varepsilon}, \gamma\left(u^{\varepsilon}\right)\right) \in L^{2}\left((0, T) ; \mathbf{V}^{\prime}\right)$, $\left(u^{\varepsilon}(0), \gamma\left(u^{\varepsilon}\right)(0)\right)=\left(u^{0}, v^{0}\right)$, such that

$\left\langle\frac{d}{d t}\left(u^{\varepsilon}, \gamma\left(u^{\varepsilon}\right)\right),(v, \gamma(v))\right\rangle+\frac{1}{\varepsilon} \int_{\Omega^{\varepsilon}} \nabla u^{\varepsilon} \cdot \nabla v d x=\frac{1}{\varepsilon} \int_{\Omega^{\varepsilon}} f v d x+\int_{\partial F^{\varepsilon}} g \gamma(v) d \sigma$,

for any $(v, \gamma(v)) \in \mathbf{V}$.

Note that here, in $H_{\partial \Omega}^{1}\left(\Omega^{\varepsilon}\right)$, we take the scalar product $\frac{1}{\varepsilon} \int_{\Omega^{\varepsilon}} \nabla u \cdot \nabla v d x$.

If we suppose that we have a better regularity of the data, we can get a more regular solution ([16]). More precisely, if the initial data $u^{0} \in H^{2}(\Omega) \cap H_{0}^{1}(\Omega)$, $v^{0}=\left.u^{0}\right|_{\partial F^{\varepsilon}}$ and $f \in C^{1}\left([0, T], L^{2}(\Omega)\right), g \in C^{1}\left([0, T], H_{0}^{1}(\Omega)\right)$, our solution satisfies $u^{\varepsilon} \in C\left([0, T] ; H^{2}\left(\Omega^{\varepsilon}\right) \cap H_{\partial \Omega}^{1}\left(\Omega^{\varepsilon}\right)\right) \cap C^{1}\left([0, T] ; L^{2}\left(\Omega^{\varepsilon}\right)\right), u_{t}^{\varepsilon} \in$ $C\left((0, T) ; H_{\partial \Omega}^{1}\left(\Omega^{\varepsilon}\right)\right)$. For such regular solutions, the weak formulation of the 
system (1.1) is the following one:

Find $u^{\varepsilon} \in C\left([0, T] ; H^{2}\left(\Omega^{\varepsilon}\right) \cap H_{\partial \Omega}^{1}\left(\Omega^{\varepsilon}\right)\right) \cap C^{1}\left([0, T] ; L^{2}\left(\Omega^{\varepsilon}\right)\right), u^{\varepsilon}(0)=\left.u^{0}\right|_{\Omega^{\varepsilon}}$ such that

$$
\begin{gathered}
-\left(u^{\varepsilon}, \frac{\partial \varphi}{\partial t}\right)_{\Omega^{\varepsilon}, T}+\left(\nabla u^{\varepsilon}, \nabla \varphi\right)_{\Omega^{\varepsilon}, T}-\varepsilon\left(u^{\varepsilon}, \frac{\partial \varphi}{\partial t}\right)_{\partial F^{\varepsilon}, T} \\
=(f, \varphi)_{\Omega^{\varepsilon}, T}+\varepsilon(g, \varphi)_{\partial F^{\varepsilon}, T}
\end{gathered}
$$

for any $\varphi \in \mathcal{D}$, or, in the other possible form

$$
\begin{array}{r}
-\int_{0}^{T}\left(u^{\varepsilon}, \varphi\right)_{\Omega^{\varepsilon}} \psi^{\prime}(s) d s-\left(u^{\varepsilon}(0), \varphi\right)_{\Omega^{\varepsilon}} \psi(0)+\int_{0}^{T}\left(\nabla u^{\varepsilon}, \nabla \varphi\right)_{\Omega^{\varepsilon}} \psi(s) d s \\
-\varepsilon \int_{0}^{T}\left(u^{\varepsilon}, \varphi\right)_{\partial F^{\varepsilon}} \psi^{\prime}(s) d s-\varepsilon\left(u^{\varepsilon}(0), \varphi\right)_{\partial F^{\varepsilon}} \psi(0) \\
=\int_{0}^{T}(f, \varphi)_{\Omega^{\varepsilon}} \psi(s) d s+\varepsilon \int_{0}^{T}(g, \varphi)_{\partial F^{\varepsilon}} \psi(s) d s
\end{array}
$$

for any $\varphi \in H_{\partial \Omega}^{1}\left(\Omega^{\varepsilon}\right), \psi \in C^{\infty}([0, T]), \psi(T)=0, \psi(0) \neq 0$.

\subsection{A Convergence Result}

For obtaining the macromodel, we have to pass to the limit, with $\varepsilon \rightarrow 0$, in some surface integrals on the boundary of the holes. For doing this, we shall make use of a convergence result based on a technique introduced by M. Vanninathan $([21])$, which transforms surface integrals into volume integrals. This method was also used for the elliptic case in [7] and [8].

For a given function $h \in L^{2}(\partial F)$, following [4], let us denote

$$
C_{h}=\frac{|\partial F|}{\left|Y^{*}\right|} \mathcal{M}_{\partial F}(h)
$$

where $\mathcal{M}_{\partial F}(h)$ is the mean value of $h$ over $\partial F$. Also, let $\mu_{h}=\theta C_{h}$. In particular $C_{1}=\frac{|\partial F|}{\left|Y^{*}\right|}$ and $\mu_{1}=\frac{|\partial F|}{|Y|}$, since $\theta=\frac{\left|Y^{*}\right|}{|Y|}$.

For $h \in L^{2}(\partial F)$, we define the measure $\mu_{h}^{\varepsilon}$ by

$$
\left\langle\mu_{h}^{\varepsilon}, \varphi\right\rangle=\varepsilon \int_{\partial F^{\varepsilon}} h\left(\frac{x}{\varepsilon}\right) \varphi(x) d \sigma, \text { for any } \varphi \in H_{0}^{1}(\Omega) .
$$

In [3] it was proved that

$$
\mu_{h}^{\varepsilon} \rightarrow \mu_{h} \text { strongly in } H^{-1}(\Omega),
$$


with $\mu_{h}=\Theta C_{h}$. Moreover, if $h$ is constant and the boundary of $F$ is smooth (of class $C^{2}$ ), the above convergence takes place strongly in $W^{-1, \infty}(\Omega)$.

In the general case, when $h \in L^{2}(\partial F)$, it was proved in [4] (Corollary 4.2) that, if $\left\{w^{\varepsilon}\right\}$ is a sequence such that $w^{\varepsilon} \in H_{0}^{1}(\Omega)$ and $w^{\varepsilon} \rightarrow w$ weakly in $H_{0}^{1}(\Omega)$, then the corresponding linear form $\mu_{h}^{\varepsilon}$ defined on $H^{1}\left(\Omega^{\varepsilon}\right)$ satisfies:

$$
\left\langle\mu_{h}^{\varepsilon}, w_{\mid \Omega^{\varepsilon}}^{\varepsilon}\right\rangle \rightarrow \mu_{h} \int_{\Omega} w d x
$$

\subsection{An Extension Lemma}

Since the solution $u^{\varepsilon}$ of the problem (1.1) is defined only in $\Omega^{\varepsilon}$, we need to extend it to the whole $\Omega$. For finding a suitable extension $\widetilde{u^{\varepsilon}}$ into all $\Omega$, we shall use the following well-known extension lemma ([9]):

Lemma 4.1. (i) Any function $\varphi \in H^{1}\left(Y^{*}\right)$ can be extended to a function $\tilde{\varphi}$ in $H^{1}(Y)$, such that

$$
\|\nabla \tilde{\varphi}\|_{Y} \leq C\|\nabla \varphi\|_{Y^{*}}
$$

(ii) Any function $\varphi^{\varepsilon} \in H^{1}\left(\Omega^{\varepsilon}\right), \varphi_{\mid \partial \Omega}^{\varepsilon}=0$ can be extended to a function $\tilde{\varphi}^{\varepsilon}$ in $H_{0}^{1}(\Omega)$, such that

$$
\left\|\nabla \tilde{\varphi}^{\varepsilon}\right\|_{\Omega} \leq C\left\|\nabla \varphi^{\varepsilon}\right\|_{\Omega^{\varepsilon}}
$$

where $C$ is a constant independent of $\varepsilon$.

\section{A PRIORI ESTIMATES. PROOF OF THE CONVERGENCE RESULT}

In this section we shall prove the convergence result given by Theorem 3.1, for the solution of the problem (1.1). This solution being defined on $\Omega^{\varepsilon}$, we need to extend it to the whole $\Omega$, in order to be able to state a convergence result. For doing this, we shall need some accurate estimates for the solution $u^{\varepsilon}$, independent of $\varepsilon$.

In what follows, we shall denote by $C$ different constants which are independent of $\varepsilon$.

Proof. [Proof of Theorem 3.1.] We shall prove first the convergence result given by Theorem 3.1 for regular initial data and so, for regular solutions $u_{n}^{\varepsilon}$. Then, by performing a classical regularization process of our initial data, we shall be able to prove immediately the convergence result for the general case.

Step 1. Regular data. Let us consider first the case in which our data is regular. In fact, let $u_{n}^{0} \in H^{2}(\Omega) \cap H_{0}^{1}(\Omega), v_{n}^{0}=\left.u_{n}^{0}\right|_{\partial \Omega^{\varepsilon}}$ such that

$$
\left(\left.u_{n}^{0}\right|_{\Omega^{\varepsilon}}, v_{n}^{0}\right) \rightarrow\left(\left.u^{0}\right|_{\Omega^{\varepsilon}}, v^{0}\right) \text { in } L^{2}\left(\Omega^{\varepsilon}\right) \times L_{\partial \Omega}^{2}\left(\partial \Omega^{\varepsilon}\right) .
$$


Also, let $f_{n} \in C^{1}\left([0, T], L^{2}(\Omega)\right), g_{n} \in C^{1}\left([0, T], H_{0}^{1}(\Omega)\right)$ such that $f_{n} \rightarrow f$ in $L^{2}\left(0, T, L^{2}(\Omega)\right)$ and $g_{n} \rightarrow g$ in $L^{2}\left(0, T, H_{0}^{1}(\Omega)\right)$.

For such regular data, for any $n$, we know that there exists a unique solution $\left(u_{n}^{\varepsilon}, \gamma\left(u_{n}^{\varepsilon}\right)\right)$ of the problem (1.1), such that

$$
\begin{aligned}
& u_{n}^{\varepsilon} \in C\left([0, T] ; H^{2}\left(\Omega^{\varepsilon}\right) \cap H_{\partial \Omega}^{1}\left(\Omega^{\varepsilon}\right)\right) \cap C^{1}\left([0, T] ; L^{2}\left(\Omega^{\varepsilon}\right)\right), \\
& \left(u_{n}^{\varepsilon}\right)_{t} \in C\left((0, T) ; H_{\partial \Omega}^{1}\left(\Omega^{\varepsilon}\right)\right) .
\end{aligned}
$$

In this step, working only with regular solutions $u_{n}^{\varepsilon}$, we shall omit to write explicitly the index $n$.

For the special geometry of our problem, we can use the following wellknown lemma, due to C. Conca ([10]):

Lemma 5.1. There exists a positive constant $C$, independent of $\varepsilon$, such that

$$
\|v\|_{\Omega^{\varepsilon}} \leq C\left(\varepsilon\|\nabla v\|_{\Omega^{\varepsilon}}+\varepsilon^{\frac{1}{2}}\|v\|_{\partial F^{\varepsilon}}\right)
$$

for any $v \in H^{1}\left(\Omega^{\varepsilon}\right), v=0$ on $\partial \Omega$.

The next proposition gives us some classical energy estimates for such a regular solution.

Proposition 5.1. For the system (1.1), the following classical parabolic estimates hold:

$$
\begin{aligned}
& \sup _{t \in(0, T)}\left(\left\|u^{\varepsilon}\right\|_{\Omega^{\varepsilon}}^{2}+\varepsilon\left\|u^{\varepsilon}\right\|_{\partial F^{\varepsilon}}^{2}\right)+\left\|\nabla u^{\varepsilon}\right\|_{\Omega^{\varepsilon}, T}^{2} \leq C, \\
& \left\|u_{t}^{\varepsilon}\right\|_{\Omega^{\varepsilon}, T}^{2}+\varepsilon\left\|u_{t}^{\varepsilon}\right\|_{\partial F^{\varepsilon}, T}^{2}+\sup _{t \in(0, T)}\left\|u^{\varepsilon}\right\|_{H_{\partial \Omega}^{1}\left(\Omega^{\varepsilon}\right)}^{2} \leq C .
\end{aligned}
$$

Proof. Let us multiply the first equation (1.1) by $u^{\varepsilon}$. We have

$$
\frac{1}{2} \frac{d}{d t}\left\|u^{\varepsilon}\right\|_{\Omega^{\varepsilon}}^{2}+\varepsilon \frac{1}{2} \frac{d}{d t}\left\|u^{\varepsilon}\right\|_{\partial F^{\varepsilon}}^{2}+\left\|\nabla u^{\varepsilon}\right\|_{\Omega^{\varepsilon}}^{2}=\int_{\Omega^{\varepsilon}} f u^{\varepsilon} d x+\varepsilon \int_{\partial F^{\varepsilon}} g u^{\varepsilon} d \sigma .
$$

Using the Cauchy-Schwartz and Young's inequalities, we get

$$
\begin{aligned}
\frac{d}{d t}\left\|u^{\varepsilon}\right\|_{\Omega^{\varepsilon}}^{2}+\varepsilon \frac{d}{d t}\left\|u^{\varepsilon}\right\|_{\partial F^{\varepsilon}}^{2} & +2\left\|\nabla u^{\varepsilon}\right\|_{\Omega^{\varepsilon}}^{2} \\
& \leq 2\|f\|_{\Omega^{\varepsilon}}\left\|u^{\varepsilon}\right\|_{\Omega^{\varepsilon}}+\varepsilon\|g\|_{\partial F^{\varepsilon}}^{2}+\varepsilon\left\|u^{\varepsilon}\right\|_{\partial F^{\varepsilon}}^{2}
\end{aligned}
$$

From the trace theorem it follows that

$$
\varepsilon\left\|u^{\varepsilon}(0)\right\|_{\partial F^{\varepsilon}}^{2} \leq C\left(\left\|u^{\varepsilon}(0)\right\|_{\Omega^{\varepsilon}}^{2}+\varepsilon^{2}\left\|\nabla u^{\varepsilon}(0)\right\|_{\Omega^{\varepsilon}}^{2}\right) .
$$


So

$$
\varepsilon\left\|u^{\varepsilon}(0)\right\|_{\partial F^{\varepsilon}}^{2} \leq C .
$$

Also

$$
\varepsilon\|g\|_{\partial F^{\varepsilon}}^{2} \leq C .
$$

Now, using Poincaré's and Young's inequalities, from (5.2), (5.4), we get

$$
\frac{d}{d t}\left\|u^{\varepsilon}\right\|_{\Omega^{\varepsilon}}^{2}+\varepsilon \frac{d}{d t}\left\|u^{\varepsilon}\right\|_{\partial F^{\varepsilon}}^{2}+\left\|\nabla u^{\varepsilon}\right\|_{\Omega^{\varepsilon}}^{2} \leq C+\varepsilon\left\|u^{\varepsilon}\right\|_{\partial F^{\varepsilon}}^{2} .
$$

Integrating in time and using Gronwall's lemma, we finally obtain

$$
\left\|u^{\varepsilon}\right\|_{\partial F^{\varepsilon}, T} \leq C \varepsilon^{-\frac{1}{2}} .
$$

Moreover, taking the supremum on $(0, T)$, from (5.2)-(5.5) we get

$$
\sup _{t \in(0, T)}\left(\left\|u^{\varepsilon}\right\|_{\Omega^{\varepsilon}}^{2}+\varepsilon\left\|u^{\varepsilon}\right\|_{\partial F^{\varepsilon}}^{2}\right)+\left\|\nabla u^{\varepsilon}\right\|_{\Omega^{\varepsilon}, T}^{2} \leq C .
$$

Let us multiply now the first equation (1.1) by $u_{t}^{\varepsilon}$. We have:

$$
\begin{aligned}
\int_{0}^{t} \int_{\Omega^{\varepsilon}}\left(u_{t}^{\varepsilon}\right)^{2}+\int_{0}^{t} \int_{\Omega^{\varepsilon}} \nabla u^{\varepsilon} \cdot \nabla u_{t}^{\varepsilon}+\varepsilon \int_{0}^{t} & \int_{\partial F^{\varepsilon}}\left(u_{t}^{\varepsilon}\right)^{2} \\
& =\int_{0}^{t} \int_{\Omega^{\varepsilon}} f u_{t}^{\varepsilon}+\varepsilon \int_{0}^{t} \int_{\partial F^{\varepsilon}} g u_{t}^{\varepsilon} .
\end{aligned}
$$

Using Young's inequality and evaluating the supremum over $(0, T)$, we get:

$$
\begin{aligned}
\left\|u_{t}^{\varepsilon}\right\|_{\Omega^{\varepsilon}, T}^{2}+\varepsilon\left\|u_{t}^{\varepsilon}\right\|_{\partial F^{\varepsilon}, T}^{2}+\sup _{t \in(0, T)}\left\|u^{\varepsilon}\right\|_{H_{\partial \Omega}^{1}\left(\Omega^{\varepsilon}\right)}^{2} & \\
& \leq\left\|u_{0}^{\varepsilon}\right\|_{H_{\partial \Omega}^{1}\left(\Omega^{\varepsilon}\right)}^{2}+\|f\|_{\Omega^{\varepsilon}, T}^{2}+\varepsilon \int_{0}^{T} \int_{\partial F^{\varepsilon}} g^{2} .
\end{aligned}
$$

But

$$
\varepsilon\|g\|_{\partial F^{\varepsilon}}^{2} \leq C .
$$

So, assuming the boundedness of our data $u^{0}, f$ and $g\left(u^{0}, f, g \neq 0\right)$, we obtain:

$$
\left\|u_{t}^{\varepsilon}\right\|_{\Omega^{\varepsilon}, T}^{2}+\varepsilon\left\|u_{t}^{\varepsilon}\right\|_{\partial F^{\varepsilon}, T}^{2}+\sup _{t \in(0, T)}\left\|u^{\varepsilon}\right\|_{H_{\partial \Omega}^{1}\left(\Omega^{\varepsilon}\right)}^{2} \leq C .
$$

In fact, one has $\left\|u^{\varepsilon}\right\|_{\mathcal{V}} \leq C$.

Using Lemma 4.1 and classical parabolic estimates, for such a regular solution, one gets 
Theorem 5.1. There exists an extension $\widetilde{u^{\varepsilon}}$ of the solution $u^{\varepsilon}$ of problem (1.1) into $\Omega$, such that

$$
\left\|\widetilde{u^{\varepsilon}}\right\|_{\Omega}+\left\|\nabla \widetilde{u^{\varepsilon}}\right\|_{\Omega, t}+\left\|\frac{\partial \widetilde{u^{\varepsilon}}}{\partial t}\right\|_{\Omega}+\left\|\frac{\partial \nabla \widetilde{u^{\varepsilon}}}{\partial t}\right\|_{\Omega, t} \leq C,
$$

for any $t \leq T$. Here, $C$ depends on the data and on $T$.

Proof. The proof follows immediately from the extension lemma and classical parabolic estimates.

Let us introduce the vector $\xi^{\varepsilon}=\chi^{\varepsilon} \tilde{\nabla u^{\varepsilon}}$. Recall that $\theta$ is the weak- $\star$ limit in $L^{\infty}(\Omega)$ of $\chi^{\varepsilon}$.

Lemma 5.2. There exist a function $u \in \mathcal{V}(u$ will be the unique solution of the limit system (3.1)) and a function $\xi \in \mathcal{H}$ such that, at least after extraction of a subsequence, we have the following convergences:

$$
\begin{aligned}
& \widetilde{u^{\varepsilon}}-u \text { weakly in } \mathcal{V} \text { and strongly in } \mathcal{H}, \\
& \frac{\partial \widetilde{u^{\varepsilon}}}{\partial t} \rightarrow \frac{\partial u}{\partial t} \text { weakly in } \mathcal{H}, \\
& \chi^{\varepsilon} \widetilde{u^{\varepsilon}}-\theta u \text { weakly in } \mathcal{H}, \\
& \xi^{\varepsilon}=\chi^{\varepsilon} \nabla \widetilde{u^{\varepsilon}}-\xi \text { weakly in } \mathcal{H}, \\
& \varepsilon\left(\frac{\partial u^{\varepsilon}}{\partial t}, \varphi\right)_{\partial F^{\varepsilon}, T} \rightarrow \frac{|\partial F|}{|Y|}\left(\frac{\partial u}{\partial t}, \varphi\right)_{\Omega, T}, \text { for all } \varphi \in \mathcal{D} \text {. }
\end{aligned}
$$

Proof. [Proof of Lemma 5.2] (5.7) and (5.8) are direct consequences of the estimates given by Proposition 5.1 and Theorem 5.1. (5.9) follows immediately from the fact that $\widetilde{u^{\varepsilon}} \rightarrow u$ strongly in $\mathcal{H}$ and $\chi^{\varepsilon} \rightarrow \theta$, weakly- $\star$ in $L^{\infty}(\Omega)$. Also, (5.10) follows from our a priori estimates. Indeed, we have $\left\|\xi^{\varepsilon}\right\|_{\Omega, T} \leq C$, and hence, up to a sequence, there exists $\xi \in \mathcal{H}$ such that $\xi^{\varepsilon} \rightarrow \xi$ weakly in $\mathcal{H}$. It remains to prove (5.11).

Let us consider a test function $\varphi \in \mathcal{D}$. It is easy to see that choosing $h=1$ and taking $w^{\varepsilon}=\widetilde{u^{\varepsilon}} \varphi_{t}$, from (4.5) we get

$$
\varepsilon \int_{\partial F^{\varepsilon}} u^{\varepsilon} \varphi_{t} d \sigma=\left\langle\mu_{1}^{\varepsilon}, \widetilde{u^{\varepsilon}} \varphi_{t \mid \Omega^{\varepsilon}}\right\rangle \rightarrow \mu_{1} \int_{\Omega} u \varphi_{t} d x=\frac{|\partial F|}{|Y|} \int_{\Omega} u \varphi_{t} d x
$$

which, integrating in time and using Lebesgue's convergence theorem, gives exactly (5.11).

Now, let us come back to the first step of the proof of Theorem 3.1. It remains only to obtain the limit equation (3.1) satisfied by $u$ and $\xi$. Let 
$\varphi \in \mathcal{D}$. We have:

$$
\begin{aligned}
& \int_{\Omega^{\varepsilon}} u^{\varepsilon} \varphi_{t} d x=\int_{\Omega} \chi^{\varepsilon} \widetilde{u^{\varepsilon}} \varphi_{t} d x \rightarrow \theta \int_{\Omega} u \varphi_{t} d x=\frac{\left|Y^{*}\right|}{|Y|} \int_{\Omega} u \varphi_{t} d x \\
& \int_{\Omega^{\varepsilon}} \nabla u^{\varepsilon} \nabla \varphi d x=\int_{\Omega} \chi^{\varepsilon} \nabla \widetilde{u^{\varepsilon}} \cdot \nabla \varphi d x \rightarrow \int_{\Omega} \xi \cdot \nabla \varphi d x \\
& \varepsilon \int_{\partial F^{\varepsilon}} g \varphi d \sigma=\left\langle\mu_{1}^{\varepsilon}, g \varphi\right\rangle \rightarrow \mu_{1} \int_{\Omega} g \varphi d x=\frac{|\partial F|}{|Y|} \int_{\Omega} g \varphi d x \\
& \int_{\Omega^{\varepsilon}} f \varphi d x=\int_{\Omega} \chi^{\varepsilon} f \varphi d x \rightarrow \theta \int_{\Omega} f \varphi d x=\frac{\left|Y^{*}\right|}{|Y|} \int_{\Omega} f \varphi d x
\end{aligned}
$$

So, all the terms in (4.3) pass to the limit, as $\varepsilon \rightarrow 0$, and, therefore, we get

$$
-\left(\frac{\left|Y^{*}\right|}{|Y|}+\frac{|\partial F|}{|Y|}\right)\left(u, \frac{\partial \varphi}{\partial t}\right)_{\Omega, T}+(\xi, \nabla \varphi)_{\Omega, T}=\frac{\left|Y^{*}\right|}{|Y|}(f, \varphi)_{\Omega, T}+\frac{|\partial F|}{|Y|}(g, \varphi)_{\Omega, T},
$$

for any $\varphi \in \mathcal{D}$. But exactly as in the elliptical case (see [7]), we have

$$
\xi_{i}=q_{i j} \frac{\partial u}{\partial x_{j}}
$$

and, finally, putting together (5.11) - (5.17) and having in mind that the solution of the macromodel is unique, the entire sequence of solutions of the microscopic model converges as necessary. So, we get (3.1). Passing to the limit with $\varepsilon \rightarrow 0$ in (4.4), we get immediately the initial condition of (3.1) and since boundary conditions of (3.1) are obviously satisfied, the proof of Theorem 3.1 for regular solutions is complete.

Step 2. The general case; density arguments. Let us prove now the convergence result given by Theorem 3.1 for the general case, in which the initial data $\left(u^{0}, v^{0}\right) \in L^{2}(\Omega) \times L_{\partial \Omega}^{2}\left(\partial \Omega^{\varepsilon}\right)$ and $f \in L^{2}\left(0, T, L^{2}(\Omega)\right), g \in L^{2}\left(0, T, H_{0}^{1}(\Omega)\right)$.

For doing this, let us consider, as already mentioned, $u_{n}^{0} \in H^{2}(\Omega) \cap H_{0}^{1}(\Omega)$, $v_{n}^{0}=\left.u_{n}^{0}\right|_{\partial \Omega^{\varepsilon}}$ such that $\left(\left.u_{n}^{0}\right|_{\Omega^{\varepsilon}}, v_{n}^{0}\right) \rightarrow\left(\left.u^{0}\right|_{\Omega^{\varepsilon}}, v^{0}\right)$ in $L^{2}\left(\Omega^{\varepsilon}\right) \times L_{\partial \Omega}^{2}\left(\partial \Omega^{\varepsilon}\right)$. Also, let $f_{n} \in C^{1}\left([0, T], L^{2}(\Omega)\right), g_{n} \in C^{1}\left([0, T], H_{0}^{1}(\Omega)\right)$ such that $f_{n} \rightarrow f$ in $L^{2}\left(0, T, L^{2}(\Omega)\right)$ and $g_{n} \rightarrow g$ in $L^{2}\left(0, T, H_{0}^{1}(\Omega)\right)$.

For any $n$, we know that

$$
\begin{aligned}
& u_{n}^{\varepsilon} \in C\left([0, T] ; H^{2}\left(\Omega^{\varepsilon}\right) \cap H_{\partial \Omega}^{1}\left(\Omega^{\varepsilon}\right)\right) \cap C^{1}\left([0, T] ; L^{2}\left(\Omega^{\varepsilon}\right)\right), \\
& \left(u_{n}^{\varepsilon}\right)_{t} \in C\left((0, T) ; H_{\partial \Omega}^{1}\left(\Omega^{\varepsilon}\right)\right) .
\end{aligned}
$$

Also, we know that all the results given by Proposition 5.1, Lemma 4.1 and Lemma 5.2 hold. So, the extension $\tilde{u_{n}^{\varepsilon}}$ converges strongly in $\mathcal{H}$ or weakly in $\mathcal{V}$ to $u_{n}$. 
The weak formulation for $u_{n}$, given by (3.4) is:

Find $u_{n} \in C\left([0, T] ; H^{2}(\Omega) \cap H_{0}^{1}(\Omega)\right) \cap C^{1}\left([0, T] ; L^{2}(\Omega)\right), u_{n}(0)=u_{n}^{0}$ such that:

$$
\begin{gathered}
-\frac{\left|Y^{*}\right|}{|Y|} \int_{0}^{T}\left(u_{n}, \varphi\right)_{\Omega} \psi^{\prime}(s) d s-\frac{\left|Y^{*}\right|}{|Y|}\left(u_{n}(0), \varphi\right)_{\Omega} \psi(0)-\frac{|\partial F|}{|Y|} \\
\times \int_{0}^{T}\left(u_{n}, \varphi\right)_{\Omega} \psi^{\prime}(s) d s-\frac{|\partial F|}{|Y|}\left(u_{n}(0), \varphi\right)_{\Omega} \psi(0)+\int_{0}^{T}\left(Q \nabla u_{n}, \nabla \varphi\right)_{\Omega} \psi(s) d s \\
=\frac{\left|Y^{*}\right|}{|Y|} \int_{0}^{T}\left(f_{n}, \varphi\right)_{\Omega} \psi(s) d s+\frac{|\partial F|}{|Y|} \int_{0}^{T}\left(g_{n}, \varphi\right)_{\Omega} \psi(s) d s
\end{gathered}
$$

for any $\varphi \in H_{0}^{1}(\Omega), \psi \in C^{\infty}([0, T]), \psi(T)=0, \psi(0) \neq 0$.

Now, by taking subsequences, if necessary, using classical energy estimates for $\left\|\widetilde{u^{\varepsilon}}-\widetilde{u_{n}^{\varepsilon}}\right\|$, we can pass to the limit in (5.18), with $n \rightarrow \infty$. We get

$$
\begin{aligned}
-\frac{\left|Y^{*}\right|}{|Y|}\left(\int_{0}^{T}(u, \varphi)_{\Omega} \psi^{\prime}(s) d s+\left(u^{0}, \varphi\right)_{\Omega} \psi(0)\right)-\frac{|\partial F|}{|Y|} \int_{0}^{T}(u, \varphi)_{\Omega} \psi^{\prime}(s) d s \\
-\frac{|\partial F|}{|Y|}\left(u^{0}, \varphi\right)_{\Omega} \psi(0)+\int_{0}^{T}(Q \nabla u, \nabla \varphi)_{\Omega} \psi(s) d s \\
=\frac{\left|Y^{*}\right|}{|Y|} \int_{0}^{T}(f, \varphi)_{\Omega} \psi(s) d s+\frac{|\partial F|}{|Y|} \int_{0}^{T}(g, \varphi)_{\Omega} \psi(s) d s
\end{aligned}
$$

for any $\varphi \in H_{0}^{1}(\Omega), \psi \in C^{\infty}([0, T]), \psi(T)=0, \psi(0) \neq 0$ and also

$$
u(0)=u^{0}, \text { in } \Omega
$$

So, we have exactly the weak formulation (3.4) of the homogenized problem and this ends the proof of the theorem.

\section{REFERENCES}

[1] G. Allaire. Homogenization and two-scale convergence. SIAM J. Math. Anal., 23, 1482 $-1518,1992$.

[2] S.N. Antontsev, A.V. Kazhikhov and V.N. Monakhov. Boundary Value Problems in Mechanics of Nonhomogeneous Fluids. North-Holland, Amsterdam, 1990.

[3] N. Bakhvalov and G. Panasenko. Homogenisation: Averaging Processes in Periodic Media, Mathematics and its Applications, volume 36. Kluwer Academic Publishers, 1989. 
[4] I. Bejenaru, J. I. Díaz and I. I. Vrabie. An abstract approximate controllability result and applications to elliptic and parabolic systems with dynamic boundary conditions. Electronic Journal of Differential Equation, 48, 1 - 19, 2001.

[5] H. Brézis. Analyse Fonctionnelle. Theorie et Applications. Masson, 1983.

[6] D. Cioranescu and P. Donato. An Introduction to Homogenization, volume 17 of Oxford Lecture Series in Mathematics and its Applications. The Clarendon Press, Oxford University Press, New York, 1999.

[7] D. Cioranescu and P. Donato. Homogénéisation du problème de Neumann non homogène dans des ouverts perforés. Asymptotic Anal., 1, 115 - 138, 1988.

[8] D. Cioranescu, P. Donato and H. Ene. Homogenization of the Stokes problem with non homogeneous slip boundary conditions. Mathematical Methods in Applied Sciences, 19, $857-881,1996$.

[9] D. Cioranescu and J. Saint Jean Paulin. Homogenization in open sets with holes. J. Math. Anal. Appl., 71, $590-607,1979$.

[10] C. Conca. On the application of the homogenization theory to a class of problems arising in fluid mechanics. J. Math. Pures Appl., 64, 31-75, 1985.

[11] C. Conca, J.I. Díaz and C. Timofte. Effective Chemical Processes in Porous Media. Math. Models Methods Appl. Sci., (M3AS), 13(10), 1437 - 1462, 2003.

[12] C. Conca and P. Donato. Non homogeneous Neumann problems in domains with small holes. RAIRO Modél. Math. Anal. Numér, 22, 561 - 607, 1988.

[13] C. Conca, F. Murat and C. Timofte. A generalized strange term in Signorini's type problems. Math. Model. Numer. Anal. (M2AN), 37(5), 773 - 806, 2003.

[14] E. De Giorgi and T. Franzoni. Su un tipo di convergenza variazionale. Atti. Accad. Naz. Lincei Rend. Cl. Sci. Mat. Fis. Natur., 58, $842-850,1975$.

[15] V. V. Jikov, S. M. Kozlov and O. A. Oleinik. Homogenization of Differential Operators and Integral Functionals. Springer-Verlag, Boston, 1994.

[16] J. L. Lions and E. Magenes. Non-Homogeneous Boundary Value Problems and Applications, volume 1. Springer-Verlag, Berlin, New York, 1982.

[17] J.L. Lions. Quelques Méthodes de Résolution des Problèmes aux Limites non Linéaires, 1969.

[18] G. Dal Maso. An Introduction to $\Gamma-$ Convergence, volume 8. Birkhäuser, Boston, 1993.

[19] E. Sanchez-Palencia. Non-Homogeneous Media and Vibration Theory. Springer-Verlag, New York, 1980.

[20] L. Tartar. Problèmes d'homogénéisation dans les équations aux dérivées partielles. In: Cours Peccot, Collège de France, 1977.

[21] M. Vanninathan. Homogenization of eigenvalues problems in perforated domains. In: Proc. Indian Acad. Sci., volume 90, 239 - 271, 1981.

[22] V.V. Zhikov. On an extension of the methd of two-scale convergence and its applications. Sbornik Mathematics, 191(7 - 8), 973 - 1014, 2000.

\section{Paraboliniai uždaviniai su dinaminėmis kraštinėmis sąlygomis per- foruotoje terpèje}

C. Timofte

Nagrinėjamas parabolinis uždavinys su dinaminemis kraštinėmis sąlygomis periodiškai perforuotoje srityje. Analizuojamos šio uždavinio sprendinio asimptotinès savybès, kai mažas parametras yra perforacijos dydis, iš mikromodelio išvedamos matematinio makromodelio lygtys ir papildomosios sąlygos. 